Georgian Mathematical Journal

Volume 13 (2006), Number 1, 25-34

\title{
FIXED POINT TECHNIQUES AND STABILITY FOR NEUTRAL NONLINEAR DIFFERENTIAL EQUATIONS WITH UNBOUNDED DELAYS
}

\author{
AHCENE DJOUDI AND RABAH KHEMIS
}

\begin{abstract}
We use the contraction mapping theorem to obtain stability results of the scalar nonlinear neutral differential equation with functional delay $x^{\prime}(t)=-a x(t)+b(t) x^{2}(t-r(t))+c(t) x(t-r(t)) x^{\prime}(t-r(t))$.
\end{abstract}

2000 Mathematics Subject Classification: 34K20, 47H10.

Key words and phrases: Contraction mapping, stability, nonlinear neutral differential equation, integral equation.

\section{InTRODUCTION}

Liapunov's method has been very effective in establishing stability results (see [2], [7] and [9]) and the existence of periodic solutions (see [2] and [8]) for a wide variety of differential equations. Nevertheless, in the applications of Liapunov's direct method to problems of stability in delay differential equations, serious difficulties occur if the functions in the equations are unbounded with time (see [7]), the delay is unbounded or the derivative of the delay is not small (Seifert [9]). In [4], when studying stability theory for ordinary and functional differential equations by means of fixed point theory, T. A. Burton and T. Furumuchi noticed that these difficulties vanish when we apply fixed point theory. In particular, in the papers [1], [3] and [5], the authors examined particular problems which have offered great difficulties for Liapunov's theory and presented solutions by means of various fixed point theories. Following these ideas, we present here a study of stability in neutral nonlinear differential equations with functional delays by means of the contraction mapping theorem.

Let us begin with a classical and simple example to show the difficulties encountered in using the Liapunov's direct method. In [6], the problem

$$
x^{\prime}(t)=-a(t) x(t)+b(t) x(t-r)
$$

is considered, where $a$ and $b$ are bounded continuous functions such that

$$
a(t) \geq \delta>0, \quad|b(t)| \leq \theta \delta, \quad \theta<1 .
$$

Consider the Liapunov functional

$$
V\left(t, x_{t}\right)=\frac{1}{2} x^{2}(t)+\delta \int_{t-r}^{t} x^{2}(s) d s .
$$


Then, using (1.1) and a triangle inequality, we obtain

$$
V^{\prime} \leq \frac{\delta}{2}(\theta-1)\left(x^{2}(t)+x^{2}(t-r)\right)
$$

Now, following the method in [2], p. 264, we conclude that the zero solution of (1.1) is uniformly asymptotically stable.

It is difficult to consider the case where $a, b$ are bounded and $|b(t)|$ is bounded by $a$ for all $t$. However we can avoid difficulties by using fixed point theory.

\section{Stability By Fixed Point Theory}

Consider the nonlinear neutral differential equation with an unbounded delay

$$
x^{\prime}(t)=-a(t) x(t)+b(t) g(x(t-r(t)))+c(t) x^{\prime}(t-r(t)),
$$

where $a(t), b(t)$ are continuous, $c(t)$ is continuously differentiable and $r(t)>0$ for all $t \in \mathbb{R}$ and is twice continuously differentiable. Suppose that there is $L>0$ such that if $|x|,|y| \leq L$, then

$$
g(0)=0 \text { and }|g(x)-g(y)| \leq|x-y| .
$$

Note that (2.1) is more complicated than (1.1) considered above since (2.1) is not linear, the delay is not constant and besides it contains a derivative term.

We have to invert equation (2.1). For this, we use the variation of parameter formula to rewrite the equation as an integral mapping equation suitable for the contraction mapping theorems. Besides, the integration by parts will be applied. We set

$$
r^{\prime}(t) \neq 1, \quad \forall t \in \mathbb{R}
$$

Lemma 1. Suppose that (2.3) holds. Then $x(t)$ is a solution of (2.1) if and only if

$$
\begin{aligned}
x(t)= & \left(x(0)-\frac{c(0)}{1-r^{\prime}(0)} x(-r(0))\right) e^{-\int_{0}^{t} a(s) d s}+\frac{c(t)}{1-r^{\prime}(t)} x(t-r(t)) \\
& -\int_{0}^{t}(h(u) x(u-r(u))-b(u) g(x(u-r(u)))) e^{-\int_{u}^{t} a(s) d s} d u,
\end{aligned}
$$

where

$$
h(u)=\frac{r^{\prime \prime}(u) c(u)+\left(c^{\prime}(u)+c(u) a(u)\right)\left(1-r^{\prime}(u)\right)}{\left(1-r^{\prime}(u)\right)^{2}} .
$$

Proof. Multiplying both side of (2.1) by $e^{\int_{0}^{t} a(s) d s}$ and then integrating from 0 to $t$, we get

$$
\int_{0}^{t}\left[x(u) e^{\int_{0}^{u} a(s) d s}\right]^{\prime} d u=\int_{0}^{t}\left[b(u) g(x(u-r(u)))+c(u) x^{\prime}(u-r(u))\right] e^{-\int_{0}^{u} a(s) d s} d u .
$$


Consequently, we have

$$
x(t) e^{\int_{0}^{t} a(s) d s}-x(0)=\int_{0}^{t}\left[b(u) g(x(u-r(u)))+c(u) x^{\prime}(u-r(u))\right] e^{-\int_{0}^{u} a(s) d s} d u .
$$

Dividing both sides of the above equation by $e^{\int_{0}^{t} a(s) d s}$, we obtain

$$
\begin{aligned}
x(t)=x(0) e^{-\int_{0}^{t} a(s) d s} & \\
& +\int_{0}^{t}\left[b(u) g(x(u-r(u)))+c(u) x^{\prime}(u-r(u))\right] e^{-\int_{u}^{t} a(s) d s} d u .
\end{aligned}
$$

Letting

$$
\begin{aligned}
& \int_{0}^{t} c(u) x^{\prime}(u-r(u)) e^{-\int_{u}^{t} a(s) d s} d u \\
= & \int_{0}^{t}\left(1-r^{\prime}(u)\right) x^{\prime}(u-r(u)) \frac{c(u)}{\left(1-r^{\prime}(u)\right)} e^{-\int_{u}^{t} a(s) d s} d u,
\end{aligned}
$$

taking

$$
U=\frac{c(u)}{\left(1-r^{\prime}(u)\right)} e^{-\int_{u}^{t} a(s) d s} \quad \text { and } \quad d V=\left(1-r^{\prime}(u)\right) x^{\prime}(u-r(u))
$$

and integrating the above integral by parts we obtain

$$
\begin{aligned}
& \int_{0}^{t} c(u) x^{\prime}(u-r(u)) e^{-\int_{u}^{t} a(s) d s} d u=\frac{c(t)}{1-r^{\prime}(t)} x(t-r(t)) \\
& \quad-\frac{c(0)}{1-r^{\prime}(0)} x(-r(0)) e^{-\int_{0}^{t} a(s) d s}-\int_{0}^{t} h(u) x(u-r(u)) e^{-\int_{u}^{t} a(s) d s} d u,
\end{aligned}
$$

where $h(u)$ is given by (2.5). Finally, substituting (2.7) into (2.6) ends the proof.

Next, let $\psi:(-\infty, 0] \rightarrow \mathbb{R}$ be a given continuous bounded initial function. We say that $x(t):=x(t, 0, \psi)$ is a solution of $(2.1)$ if $x(t)=\psi(t)$ for $t \leq 0$ and satisfies (2.1) for $t \geq 0$. Let $C$ be the space of all continuous functions from $\mathbb{R} \rightarrow \mathbb{R}$ and define the set $S_{\psi}$ by

$$
\begin{gathered}
S_{\psi}=\{\varphi: \mathbb{R} \rightarrow \mathbb{R} /\|\varphi\| \leq L, \quad \varphi(t)=\psi(t) \text { if } t \leq 0, \\
\varphi(t) \rightarrow 0 \text { as } t \rightarrow \infty, \varphi \in C\} .
\end{gathered}
$$

Then, equipped with the supremum norm \|\|$, S_{\psi}$ is a Banach space.

For the next theorem we need the conditions:

$$
e^{-\int_{0}^{t} a(s) d s} \rightarrow 0 \quad \text { as } \quad t \rightarrow \infty
$$


there is $\alpha>0$ such that $\alpha<1$,

$$
\left|\frac{c(t)}{1-r^{\prime}(t)}\right|+\int_{0}^{t}(|h(u)|+|b(u)|) e^{-\int_{u}^{t} a(s) d s} d u \leq \alpha, \quad t \geq 0,
$$

and

$$
t-r(t) \rightarrow \infty \quad \text { as } \quad t \rightarrow \infty
$$

Theorem 2. If (2.2) and (2.8)-(2.10) hold, then every solution $x(t, 0, \psi)$ of (2.1) with a small continuous initial function $\psi(t)$ is bounded and tends to zero as $t \rightarrow \infty$. Moreover, the zero solution is stable at $t_{0}=0$.

Proof. For $\alpha$ and $L$, find an appropriate $\delta>0$ such that

$$
\left|1-\frac{c(0)}{1-r^{\prime}(0)}\right| \delta+\alpha L \leq L
$$

Let $\psi:(-\infty, 0] \rightarrow \mathbb{R}$ be a given small bounded initial function with $\|\psi\|<\delta$. Define the mapping $P: S_{\psi} \rightarrow S_{\psi}$ by

$$
\begin{aligned}
(P \varphi)(t) & =\left(\varphi(0)-\frac{c(0)}{1-r^{\prime}(0)} \varphi(-r(0))\right) e^{-\int_{0}^{t} a(s) d s}+\frac{c(t)}{1-r^{\prime}(t)} \varphi(t-r(t)) \\
& -\int_{0}^{t}(h(u) \varphi(u-r(u))-b(u) g(\varphi(u-r(u)))) e^{-\int_{u}^{t} a(s) d s} d u, \quad t \geq 0 .
\end{aligned}
$$

Clearly, $P \varphi$ is continuous when $\varphi$ is such. Let $\varphi \in S_{\psi}$, then, using (2.9) in the definition of $(P \varphi)(t)$ and applying $(2.2)$, we have

$$
\begin{aligned}
& |(P \varphi)(t)| \leq\left|1-\frac{c(0)}{1-r^{\prime}(0)}\right| \delta+\left|\frac{c(t)}{1-r^{\prime}(t)}\right| L \\
& \quad+\int_{0}^{t}(|h(u)||\varphi(u-r(u))|+|b(u)||g(\varphi(u-r(u)))|) e^{-\int_{u}^{t} a(s) d s} d u \\
& \leq\left|1-\frac{c(0)}{1-r^{\prime}(0)}\right| \delta+\left|\frac{c(t)}{1-r^{\prime}(t)}\right| L \\
& \quad+\int_{0}^{t}(|h(u)|+|b(u)|)|\varphi(u-r(u))| e^{-\int_{u}^{t} a(s) d s} d u \\
& \leq\left|1-\frac{c(0)}{1-r^{\prime}(0)}\right| \delta+L\left\{\left|\frac{c(t)}{1-r^{\prime}(t)}\right|+\int_{0}^{t}(|h(u)|+|b(u)|) e^{-\int_{u}^{t} a(s) d s} d u\right\} \\
& \leq\left|1-\frac{c(0)}{1-r^{\prime}(0)}\right| \delta+L \alpha
\end{aligned}
$$

which implies that $|(P \varphi)(t)| \leq L$ for the chosen $\delta$. Thus we have $\|P \varphi\| \leq L$. Next we show that $(P \varphi)(t) \rightarrow 0$ as $t \rightarrow \infty$. By condition $(2.8)$, the first term in 
the definition of $(P \varphi)(t)$ tends to zero. Also, the second term on the right-hand side tends to zero because of $(2.10)$ and the fact that $\varphi \in S_{\psi}$. It remains to show that the integral term tends to zero as $t \rightarrow \infty$.

Let $\varepsilon>0$ be arbitrary and $\varphi \in S_{\psi}$. Then $\|\varphi\| \leq L$ and there exists $t_{1}>0$ such that $|\varphi(t-r(t))|<\varepsilon$ for $t \geq t_{1}$. By condition (2.8), there exists $t_{2}>t_{1}$ such that for $t>t_{2}$

Thus, for $t>t_{2}$, we have

$$
e^{-\int_{t_{1}}^{t} a(s) d s}<\frac{\varepsilon}{\alpha L}
$$

$$
\begin{aligned}
& \left|\int_{0}^{t}(h(u) \varphi(u-r(u))-b(u) g(\varphi(u-r(u)))) e^{-\int_{u}^{t} a(s) d s} d u\right| \\
& \quad \leq \int_{0}^{t}(|h(u)||\varphi(u-r(u))|+|b(u)||g(\varphi(u-r(u)))|) e^{-\int_{u}^{t} a(s) d s} d u \\
& \quad \leq \int_{0}^{t}(|h(u)|+|b(u)|)|\varphi(u-r(u))| e^{-\int_{u}^{t} a(s) d s} d u \\
& \quad \leq L \int_{0}^{t_{1}}(|h(u)|+|b(u)|) e^{-\int_{u}^{t} a(s) d s} d u+\varepsilon \int_{t_{1}}^{t}(|h(u)|+|b(u)|) e^{-\int_{u}^{t} a(s) d s} d u \\
& \leq L e^{-\int_{t_{1}}^{t} a(s) d s} \int_{0}^{t_{1}}(|h(u)|+|b(u)|) e^{-\int_{u}^{t_{1}} a(s) d s} d u+\alpha \varepsilon \\
& \leq \alpha L e^{-\int_{t_{1}}^{t} a(s) d s}+\alpha \varepsilon \leq \varepsilon+\alpha \varepsilon .
\end{aligned}
$$

Hence $(P \varphi)(t) \rightarrow 0$ as $t \rightarrow \infty$.

It remains to show that $(P \varphi)$ is a contraction under the supremum norm. For this, let $\varphi, \phi \in S$. Then

$$
\begin{aligned}
& |(P \varphi)(t)-(P \phi)(t)| \leq\left|\frac{c(t)}{1-r^{\prime}(t)}\right|\|\varphi-\phi\| \\
& \quad+\int_{0}^{t}|h(u)(\varphi(u-r(u))-\phi(u-r(u)))| e^{-\int_{u}^{t} a(s) d s} d u \\
& \quad+\int_{0}^{t}|b(u)(g(\varphi(u-r(u)))-g(\phi(u-r(u))))| e^{-\int_{u}^{t} a(s) d s} d u \\
& \leq\left\{\left|\frac{c(t)}{1-r^{\prime}(t)}\right|+\int_{0}^{t}(|h(u)|+|b(u)|) e^{-\int_{u}^{t} a(s) d s} d u\right\}\|\varphi-\phi\| \\
& \leq \alpha\|\varphi-\phi\| .
\end{aligned}
$$


Thus, by the contraction mapping principle, $P$ has a unique fixed point in $S_{\psi}$ which solves (2.1), is bounded and tends to zero as $t \rightarrow \infty$. To obtain the stability of the trivial solution at $t_{0}=0$, we let $\varepsilon>0$ be arbitrary and we repeat the above reasoning where $L$ is replacing by $\varepsilon$.

Now we turn our attention to the following nonlinear neutral differential equation with an unbounded delay:

$$
x^{\prime}(t)=-a(t) x(t)+b(t) x^{2}(t-r(t))+c(t) x(t-r(t)) x^{\prime}(t-r(t)) .
$$

We use the variation of parameter to get the solution

$$
\begin{aligned}
x(t)= & x(0) e^{-\int_{0}^{t} a(s) d s} \\
& +\int_{0}^{t}\left(c(u) x(u-r(u)) x^{\prime}(u-r(u))+b(u) x^{2}(u-r(u))\right) e^{-\int_{u}^{t} a(s) d s} d u .
\end{aligned}
$$

Taking

$$
\begin{aligned}
& \int_{0}^{t} c(u) x(u-r(u)) x^{\prime}(u-r(u)) e^{-\int_{u}^{t} a(s) d s} d u \\
& =\int_{0}^{t} x(u-r(u)) x^{\prime}(u-r(u))\left(1-r^{\prime}(u) \frac{c(u)}{\left(1-r^{\prime}(u)\right.} e^{-\int_{u}^{t} a(s) d s} d u\right.
\end{aligned}
$$

and integrating by parts the right-hand side of this equation with

$$
U=\frac{c(u)}{\left(1-r^{\prime}(u)\right.} e^{-\int_{u}^{t} a(s) d s}
$$

and

$$
d V=x(u-r(u)) x^{\prime}(u-r(u))\left(1-r^{\prime}(u),\right.
$$

we obtain

$$
\begin{aligned}
\int_{0}^{t} x(u-r(u)) x^{\prime}(u-r(u))\left(1-r^{\prime}(u) \frac{c(u)}{\left(1-r^{\prime}(u)\right.} e^{-\int_{u}^{t} a(s) d s} d u\right. & \\
=\frac{1}{2} x^{2}(t-r(t)) \frac{c(t)}{1-r^{\prime}(t)}- & \frac{1}{2} x^{2}(-r(0)) \frac{c(0)}{1-r^{\prime}(0)} e^{-\int_{0}^{t} a(s) d s} \\
& -\frac{1}{2} \int_{0}^{t} h(u) x^{2}(u-r(u)) e^{-\int_{u}^{t} a(s) d s} d u
\end{aligned}
$$

where $h(u)$ is given by (2.5). Thus a solution of (2.11) has the form

$$
x(t)=\left(x(0)-\frac{1}{2} \frac{c(0)}{1-r^{\prime}(0)} x^{2}(-r(0))\right) e^{-\int_{0}^{t} a(s) d s}
$$




$$
+\frac{1}{2} x^{2}(t-r(t)) \frac{c(t)}{1-r^{\prime}(t)}-\frac{1}{2} \int_{0}^{t}(h(u)-2 b(u)) x^{2}(u-r(u)) e^{-\int_{u}^{t} a(s) d s} d u .
$$

Let

$$
\begin{gathered}
S_{\psi}=\{\varphi: \mathbb{R} \rightarrow R /\|\varphi\| \leq L, \quad \varphi(t)=\psi(t) \text { if } t \leq 0 \\
\varphi(t) \rightarrow 0 \text { as } t \rightarrow \infty, \varphi \in C\} .
\end{gathered}
$$

Suppose that

$$
L\left\{\left|\frac{c(t)}{1-r^{\prime}(t)}\right|+\int_{0}^{t}|h(u)-2 b(u)| e^{-\int_{u}^{t} a(s) d s} d u\right\} \leq \alpha<1, \quad t \geq 0 .
$$

Theorem 3. If (2.8), (2.10) and (2.12) hold, then every solution $x(t, 0, \psi)$ of (2.11), with a small continuous initial function $\psi(t)$, is bounded and goes to zero as $t \rightarrow \infty$. Moreover the zero solution is stable at $t_{0}=0$.

Proof. For $L$ and $\alpha$, find $\delta>0$ such that

$$
\left(\delta+\frac{1}{2}\left|\frac{c(0)}{1-r^{\prime}(0)}\right| \delta^{2}\right)+\frac{1}{2} L \alpha \leq L .
$$

Let $\psi:(-\infty, 0] \rightarrow \mathbb{R}$ be a given small bounded initial function with $\|\psi\|<\delta$. Define the mapping $P: S_{\psi} \rightarrow S_{\psi}$ by

$$
\begin{aligned}
(P \varphi)(t)=(\varphi(0)- & \left.\frac{1}{2} \frac{c(0)}{1-r^{\prime}(0)} \varphi^{2}(-r(0))\right) e^{-\int_{0}^{t} a(s) d s}+\frac{1}{2} \frac{c(t)}{1-r^{\prime}(t)} \varphi^{2}(t-r(t)) \\
& -\frac{1}{2} \int_{0}^{t}(h(u)-2 b(u)) \varphi^{2}(u-r(u)) e^{-\int_{u}^{t} a(s) d s} d u, \quad t \geq 0 .
\end{aligned}
$$

Clearly, $P \varphi$ is continuous if $\varphi$ is such. Let $\varphi \in S_{\psi}$ with $\|\varphi\| \leq L$. Then using $(2.12)$ in the definition of $(P \varphi)(t)$, we obtain

$$
\begin{aligned}
|(P \varphi)(t)| \leq & \left(\delta+\frac{1}{2}\left|\frac{c(0)}{1-r^{\prime}(0)}\right| \delta^{2}\right) \\
& +\frac{1}{2} L^{2}\left\{\left|\frac{c(t)}{1-r^{\prime}(t)}\right|+\int_{0}^{t}|h(u)-2 b(u)| e^{-\int_{u}^{t} a(s) d s} d u\right\} \\
\leq & \left(\delta+\frac{1}{2}\left|\frac{c(0)}{1-r^{\prime}(0)}\right| \delta^{2}\right)+\frac{1}{2} L \alpha .
\end{aligned}
$$

Thus $\|(P \varphi)\| \leq L$ by choosing $\delta$ as above, and $(P \varphi)(t)$ is bounded. Consider $t_{1}$ and $t_{2}$ as in the proof of Theorem 2. Then for $t>t_{2}$, we have

$$
|(P \varphi)(t)|=\left(\delta+\frac{1}{2}\left|\frac{c(0)}{1-r^{\prime}(0)}\right| \delta^{2}\right) e^{-\int_{0}^{t} a(s) d s}+\frac{1}{2}\left|\frac{c(t)}{1-r^{\prime}(t)}\right|\left|\varphi^{2}(t-r(t))\right|
$$




$$
\begin{aligned}
& +\frac{1}{2} \int_{0}^{t}\left|(h(u)-2 b(u)) \varphi^{2}(u-r(u))\right| e^{-\int_{u}^{t} a(s) d s} d u \\
\leq & \left(\delta+\frac{1}{2}\left|\frac{c(0)}{1-r^{\prime}(0)}\right| \delta^{2}\right) \varepsilon+\frac{1}{2}\left|\frac{c(t)}{1-r^{\prime}(t)}\right| \varepsilon^{2} \\
& +\frac{1}{2} L^{2} \varepsilon \int_{0}^{t_{1}}|h(u)-2 b(u)| e^{-\int_{u}^{t_{1}} a(s) d s} d u+\frac{1}{2} \varepsilon^{2} \int_{t_{1}}^{t}|h(u)-2 b(u)| e^{-\int_{u}^{t} a(s) d s} d u \\
\leq & \left(\delta+\frac{1}{2}\left|\frac{c(0)}{1-r^{\prime}(0)}\right| \delta^{2}\right) \varepsilon+\frac{\alpha \varepsilon^{2}}{2 L}+\frac{\alpha \varepsilon L}{2} .
\end{aligned}
$$

This implies that $(P \varphi)(t) \rightarrow 0$ as $t \rightarrow 0$. To see that $P$ is contraction under the supremum norm, let $\varphi, \phi \in S_{\psi}$. Then

$$
\begin{aligned}
& |(P \varphi)(t)-(P \phi)(t)| \\
& \quad \leq \frac{1}{2}\left\{\left|\frac{c(t)}{1-r^{\prime}(t)}\right|+\int_{0}^{t}|h(u)-2 b(u)| e^{-\int_{u}^{t} a(s) d s} d u\right\}\left\|\varphi^{2}-\phi^{2}\right\| \\
& \quad \leq \frac{1}{2}(2 L)\left\{\left|\frac{c(t)}{1-r^{\prime}(t)}\right|+\int_{0}^{t}|h(u)-2 b(u)| e^{-\int_{u}^{t} a(s) d s} d u\right\}\|\varphi-\phi\| \\
& \quad \leq \alpha\|\varphi-\phi\| .
\end{aligned}
$$

The Banach theorem implies that $P$ has a unique fixed point in $S_{\psi}$ which solves (2.11), is bounded and tends to zero as $t \rightarrow \infty$. Thus we obtain the stability of the trivial solution at $t_{0}=0$.

\section{Neutral Volterra integral equation}

Consider now the scalar neutral Volterra integral equation

$$
x^{\prime}(t)=-a(t) x(t)+c(t) x^{\prime}(t-r(t)) x\left(t-r(t)+\int_{t-r(t)}^{t} K(t, s) x^{2}(s) d s,\right.
$$

where $0 \leq r(t) \leq r_{0}$ for some constant $r_{0}$.

If (2.3) holds, then a solution of (3.1) is given by

$$
\begin{aligned}
x(t)=[x(0) & \left.-\frac{1}{2} x^{2}(-r(0)) \frac{c(0)}{1-r^{\prime}(0)}\right] e^{-\int_{0}^{t} a(s) d s}+\frac{1}{2} x^{2}(t-r(t)) \frac{c(t)}{1-r^{\prime}(t)} \\
- & \left.\frac{1}{2} \int_{0}^{t}\left[h(u) x^{2}(u-r(u))\right)-\int_{u-r(u)}^{u} K(u, s) x^{2}(s) d s\right] e^{-\int_{u}^{t} a(s) d s} d u,
\end{aligned}
$$

where $h(u)$ is defined by $(2.5)$. 
Let $L$ be some positive constant and suppose that the followings conditions are satisfied

$$
L\left\{\left|\frac{c(t)}{1-r^{\prime}(t)}\right|+\int_{0}^{t}\left[|h(u)|+\int_{u-r(u)}^{u}|K(u, s)| d s\right] e^{-\int_{u}^{t} a(s) d s} d u\right\} \leq \alpha<1, t \geq 0,
$$

and that for every $\varepsilon>0$ there exists $t_{1}>0$ and $T>0$ such that $t_{2} \geq t_{1}$ and $t \geq t_{2}+T$, then we have

$$
e^{-\int_{t_{2}}^{t} a(s) d s}<\varepsilon \quad \text { and } \quad e^{-\int_{0}^{t} a(s) d s} \rightarrow 0 \quad \text { as } \quad t \rightarrow \infty .
$$

In [4], an equation similar to (3.1) is considered with $c(t)=0$.

Theorem 4. If conditions (2.3), (2.10), (3.2) and (3.3) hold, then the trivial solution of (3.1) is asymptotically stable at $t_{0}=0$.

Proof. Choose a positive number $\delta$ as in the proof of Theorem 3 and let $\psi$ : $\left[-r_{0}, 0\right] \rightarrow \mathbb{R}$ be a given bounded initial function with $\|\psi\|<\delta$. Let

$$
\begin{gathered}
S_{\psi}=\left\{\varphi:\left[-r_{0}, \infty\right] \rightarrow \mathbb{R} / \varphi(t)=\psi(t) \text { if }-r_{0} \leq t \leq 0,\|\varphi\| \leq L,\right. \\
\varphi \in C, \text { and } \varphi(t) \rightarrow 0 \text { as } t \rightarrow \infty\},
\end{gathered}
$$

where $\|\cdot\|$ is the supremum norm.

Define the mapping $P: S_{\psi} \rightarrow S_{\psi}$ by

$$
\begin{gathered}
(P \varphi)(t)=\left[\varphi(0)-\frac{1}{2} \varphi^{2}(-r(0)) \frac{c(0)}{1-r^{\prime}(0)}\right] e^{-\int_{0}^{t} a(s) d s}+\frac{1}{2} \varphi^{2}(t-r(t)) \frac{c(t)}{1-r^{\prime}(t)} \\
\left.-\frac{1}{2} \int_{0}^{t}\left[h(u) \varphi^{2}(u-r(u))\right)-\int_{u-r(u)}^{u} K(u, s) \varphi^{2}(s) d s\right] e^{-\int_{u}^{t} a(s) d s} d u, \quad t \geq 0 .
\end{gathered}
$$

Clearly, $P$ is well defined from $S_{\psi}$ to $S_{\psi}$. Let $\varphi \in S_{\psi}$ be such that $\|\varphi\| \leq L$. For any $\varepsilon>0$ there exists $t_{1}>0$ such that for $t \geq t_{1}-r_{0}$ we have $|\varphi(t)|<\varepsilon$. Also, for $t_{2} \geq t_{1}$ and $t \geq t_{2}+T$ we have $e^{-\int_{t_{2}}^{t} a(s) d s}<\varepsilon$. Consequently, $t \geq t_{2}+T$ and $t_{1}$ large enough implies

$$
\begin{aligned}
|(P \varphi)(t)| \leq & \left(\delta+\frac{1}{2}\left|\frac{c(0)}{1-r^{\prime}(0)}\right| \delta^{2}\right) \varepsilon+\frac{1}{2}\left|\frac{c(t)}{1-r^{\prime}(t)}\right| \varepsilon^{2} \\
& +\frac{1}{2} L^{2} \int_{0}^{t_{2}}\left[|h(u)|+\int_{u-r(u)}^{u}|K(u, s)| d s\right] e^{-\int_{u}^{t_{2}} a(s) d s} e^{-\int_{t_{2}}^{t} a(s) d s} d u \\
& +\frac{1}{2} \varepsilon^{2} \int_{t_{2}}^{t}\left[|h(u)|+\int_{u-r(u)}^{u}|K(u, s)| d s\right] e^{-\int_{u}^{t} a(s) d s} d u \\
\leq & \left(\delta+\frac{1}{2}\left|\frac{c(0)}{1-r^{\prime}(0)}\right| \delta^{2}\right) \varepsilon+\frac{\alpha \varepsilon^{2}}{2 L}+\frac{\alpha \varepsilon L}{2} .
\end{aligned}
$$


That is, $(P \varphi)(t) \rightarrow 0$ as $t \rightarrow \infty$. Also, $\|\varphi\| \leq L$ implies $\|P \varphi\| \leq L$ by a choice of $\delta$. We will show that $P$ is a contraction. For this, let $\varphi, \phi \in S_{\psi}$. Then (3.2) implies

$$
\begin{aligned}
& |(P \varphi)(t)-(P \phi)(t)| \\
& \leq \frac{1}{2}(2 L)\left\{\left|\frac{c(t)}{1-r^{\prime}(t)}\right|+\int_{0}^{t}\left[|h(u)|+\int_{u-r(u)}^{u}|K(u, s)| d s\right] e^{-\int_{u}^{t} a(s) d s} d u\right\}\|\varphi-\phi\| \\
& \leq \alpha\|\varphi-\phi\| .
\end{aligned}
$$

Hence $P$ has a unique fixed point in $S_{\psi}$.

\section{ACKNOWLEDGEMENT}

The authors would like to thank the referee for valuable hints

\section{REFERENCES}

1. T. A. Burton, Liapunov functionals, fixed points, and stability by Krasnoselskii's theorem. Nonlinear Stud. 9(2002), No. 2, 181-190.

2. T. A. Burton, Stability and periodic solutions of ordinary and functional-differential equations. Mathematics in Science and Engineering, 178. Academic Press, Inc., Orlando, $F L, 1985$.

3. T. A. BurTon,, Stability by fixed point theory or Liapunov theory: a comparison. Fixed Point Theory 4(2003), No. 1, 15-32.

4. T. A. Burton and T. Furumochi, Fixed points and problems in stability theory for ordinary and functional differential equations. Dynam. Systems Appl. 10(2001), No. 1, 89-116.

5. T. A. Burton and T. Furumochi, Krasnoselskii's fixed point theorem and stability. Nonlinear Anal. 49(2002), No. 4, Ser. A: Theory Methods, 445-454.

6. J. K. Hale, Theory of functional differential equations. Second edition. Applied Mathematical Sciences, Vol. 3. Springer-Verlag, New York-Heidelberg, 1977.

7. L. Hatvani, Annulus arguments in the stability theory for functional differential equations. Differential Integral Equations 10(1997), No. 5, 975-1002.

8. Y. N. RAFfoul, Periodic solutions for neutral nonlinear differential equations with functional delay. Electron. J. Differential Equations 2003, No. 102, 7 pp. (electronic).

9. G. SEIFERT, Liapunov-Razumikhin conditions for stability and boundedness of functional differential equations of Volterra type. J. Differential Equations 14(1973), 424-430.

(Received 7.03.2005)

Authors' address:

Institut de Mathématiques

Faculté des Sciences

Université Badji Mokhtar de Annaba

P.O. Box 12, 23000 Annaba

Algeria

E-Mail: adjoudi@yahoo.com 